\title{
Structure and function of the kidney in septic shock - a prospective controlled study
}

\author{
MJ Maiden ${ }^{1,2^{*}}$, S Otto $^{3}$, J Brearly ${ }^{3}$, MJ Chapman ${ }^{1,2}, \mathrm{CH} \mathrm{Nash}^{2}$, J Edwards ${ }^{1}$, TR Kuchel $^{4}$, R Bellomo $^{5}$ \\ From ESICM LIVES 2015 \\ Berlin, Germany. 3-7 October 2015
}

\section{Introduction}

Sepsis is the most common cause of acute renal failure. Impaired renal blood flow, acute tubular necrosis, glomerular micro-thrombi and apoptosis have all been proposed to cause this renal dysfunction [1,2]. However, these concepts have been largely based on uncontrolled, observational and post-mortem studies.

\section{Objectives}

Determine changes in renal structure and function over time in an ovine model of septic shock managed with intensive care support.

\section{Methods}

Fifteen sheep had renal artery flow probes and renal vein cannula inserted. Ten sheep were given intravenous E. coli to induce sepsis and five acted as non-septic controls. Animals were managed for 48 hours with protocol guided ventilation, sedation, parenteral fluids and noradrenaline (NorA) infusion to maintain mean arterial pressure $75 \mathrm{mmHg}$. Renal biopsies were taken from all animals at baseline, 24 and 48 hours or if oliguric for more than two hours. Biopsies were analysed under light and electron microscopy and appearance systematically quantified by expert renal pathologists blinded to group allocation and time of biopsy.

\section{Results}

Sheep given E. coli developed hyperdynamic septic shock requiring NorA infusion. Renal blood flow was not different between septic and non-septic groups over the duration of the study (mean $\pm \mathrm{SD}, \mathrm{mL} / \mathrm{min}$; nonseptic $428 \pm 102$ vs. septic $386 \pm 118 ; \mathrm{p}=0.12$ ). Septic sheep became oliguric and after 48 hours had increased serum creatinine (mmol/L; non-septic $69 \pm 14$ vs. septic
$200 \pm 86 ; \mathrm{p}<0.01)$ and reduced creatinine clearance $(\mathrm{mL} / \mathrm{min}$; non-septic $170 \pm 44$ vs. septic $40 \pm 40 ; \mathrm{p}<$ $0.01)$. There was no difference in mean renal $\mathrm{O}_{2}$ consumption $(\mathrm{mL} / \mathrm{min}$; non-septic $4.9 \pm 2.1$ vs. septic $5.6 \pm$ $2.3 ; \mathrm{p}=0.39$ ). Histopathological and ultrastructural appearance of each component of the nephron did not change over time and was not different between the septic and non-septic group of animals.

\section{Conclusions}

In this ovine model of Gram-negative septic shock, acute renal dysfunction was not associated with changes in renal blood flow, $\mathrm{O}_{2}$ consumption, cellular appearance or ultrastructural change. Other mechanisms are likely to contribute to the acute changes of renal function in sepsis.

\section{Grant Acknowledgment}

Intensive Care Foundation University of Adelaide, Maurice Sando Research Grant.

\section{Authors' details}

${ }^{1}$ Royal Adelaide Hospital, Intensive Care Unit, Adelaide, Australia. ${ }^{2}$ University of Adelaide, Acute Care Medicine, Adelaide, Australia. ${ }^{3}$ SA Pathology, Adelaide, Australia. ${ }^{4}$ SAHMRI, PIRL, Adelaide, Australia. ${ }^{5}$ Austin Hospital, Intensive Care Unit, Heidelberg, Australia.

\section{Published: 1 October 2015}

\section{References}

1. Lameire N, Van Biesen W, Vanholder R: Acute renal failure. Lancet 2005, 365(9457):417-430.

2. Schrier RW, Wang W: Acute renal failure and sepsis. N Engl J Med 2004, 351(2):159-169.

doi:10.1186/2197-425X-3-S1-A838

Cite this article as: Maiden et al:: Structure and function of the kidney in septic shock - a prospective controlled study. Intensive Care Medicine Experimental 2015 3(Suppl 1):A838. 